\title{
Estimating Subjective Assessments using a Simple Biosignal Sensor
}

\author{
Yoshihito Maki \\ Nagoya Instititute of Technology \\ Email:maki@ai.nitech.ac.jp
}

\author{
Genma Sano \\ Nagoya Instititute of Technology \\ Email: sano@ai.nitech.ac.jp
}

\author{
Yusuke Kobashi \\ Nagoya Instititute of Technology \\ Email:kobashi@ai.nitech.ac.jp
}

\author{
Tsuyoshi Nakamura \\ Nagoya Instititute of Technology \\ Email: tnaka@nitech.ac.jp
}

\author{
Masayoshi Kanoh \\ Chukyo University \\ Email: mkanoh@sist.chukyo-u.ac.jp
}

\author{
Koji Yamada \\ Institute of Advanced Media Arts and Sciences \\ Email: k-yamada@iamas.ac.jp
}

\begin{abstract}
Given the remarkable recent progress in robotics research, we can envision the day when robots and humans coexist and robots become closely integrated into our daily lives. This means endowing robots with the ability to communicate so they perceive human emotion, adapt their behavior to humans, and sense situations even without explicit instructions. Meanwhile, affective computing, that interprets emotion or other affective phenomena from human biosignals, has emerged as an area of great interest. In addition to biosignals-brain waves, heart rate, pulse, electrical activity, and the like-affective computing is concerned with facial expressions, gestures, and a wide range of other indicators of emotion. Here we explore the latest insights of affective computing in relation to human-robot interaction (HRI). There is good reason to believe robots will soon have the ability to read human emotions, so here we investigate the feasibility of inferring human psychological states from biosensor signals. Obviously, non-invasive biosensors that don't interfere with normal everyday activities would be preferable. A number of inexpensive user-friendly brain-wave sensors have been brought to market recently, and we employ one of these devices, the NeuroSky Mindset EEG neuroheadset, in assessment trials to explore the feasibility of inferring subjective assessments. Using our experimental setup, we find that it is indeed possible to infer subjective assessments from biosignals, and this capability could prove immensely useful for future HRI applications.
\end{abstract}

\section{INTRODUCTION}

Given the remarkable recent $R \& D$ advances in robot-related research, we can envision the day in the not-too-distant future when robots will be integrated and actively involved in everyday lives of humans. This being so, we must also assume the ability of robots to communicate with humans and support interpersonal communications. This communication must be as barrier-free and unimpeded as communication between people. To achieve this level of fluency, R\&D must focus on endowing robots with the ability to grasp the moods, emotions, and mental states of humans.

We know that in ordinary human communication, people have the ability to detect psychological shifts in the person they are talking to and to interact appropriately by adapting their own behavior and conversation to changes in the other person's mental state. If robots could be endowed with this same capability to detect and adapt to subtle changes in human psychology, this could open the way to rich and natural human-robot interaction that is similar to ordinary human communication.

It would be very difficult at this point to address the full complexity and diversity of human psychology, so our more modest objective is to consider whether a subjective face scale assessment index can be used to accurately capture the mood of subjects. More specifically, this project will investigate the extent that face scale changes reflecting subjective changes in mood correlate with estimates derived from objective observable data. This research could open the way for robots to communicate by first observing changes in human psychology or mood, than using this information to sense or read the situation and conduct themselves in ways that are helpful or solicitous to humans.

Recently the interdisciplinary field of affective computing, the study and development of systems and devices that can recognize, interpret, and simulate the emotional states of human, has attracted enormous interest [1], [2]. The range of data available for extrapolating human emotion includes not only brain-wave or electrocardiogram (EEG) patterns, electrocardiographic (ECG) patterns, and electromyogram (EMG) patterns, but also the voice, facial expressions, posture, and behavior. In choosing which type of data to observe, facial expression and speech have obvious advantages since this data can be obtained by contactless sensors, and thus imposes little burden on the user. But to achieve greater accuracy, we would expect data robustness to be enhanced by using EEG or EMG sensors that are in contact with the body. Among the various types of data and methods that are available, we wanted the accuracy that can be obtained with contact sensors yet we did not want to be overly dependent on the measurement environment, so we focused on brain-wave (EEG) biosignals. Besides brain-wave signals that we use in this study, there are various other kinds of biosignals captured by contact sensorselectrocardiogram (ECG), electromyogram (EMG), electrodermogram (EDG), and so on-but brain waves directly reflect activities of the brain, which should also include information relating to the state of human emotion.

Measuring brain waves in the past was a cumbersome procedure. It involved an enormous expensive piece of equipment 
and numerous electrodes were attached to the subject's skull with copious amounts of gel. While this approach is still in widespread use, we've recently seen the emergence of a much simpler brain-wave sensor that uses dry (i.e., no gel) electrodes. These simpler sensors support mobile applications, and if they can be further downsized, one can easily imagine a wearable implementation that could be conveniently worn throughout the day.

For this study, we used one of these new brain-wave sensors, the Mindset headphone manufactured by Neurosky, to see if we could estimate subjective assessment values based on brain-wave biosignals detected by the neuroheadset. In other words, we will explore the relationship between perceived subjective face scale assessments and biosignals captured by Mindset.

\section{Estimating Subjective Assessments}

In evaluating subjective assessments, it is well known that certain substances found in saliva-immunoglobulin A, cortisol, and so on-are indicators of stress [3]. Also well-known is the heart-rate variability analysis method. For example, Yokoyama et al. proposed a simplified scheme for estimating high-frequency components of heart rate variability called the Turning Point Amplitude (TPA) that correlates very highly with conventional AR and wavelet transformation analyses [4]. TPA can be calculated from as few as 20-30 data points, and since the calculation formula itself is relatively simple, this approach seems well suited for real-time processing. Another study based on heart-rate variability analysis, uses finger plethysmography to investigate the relationship between observable data and the subjective assessments of subjects (similar to the face scale scheme used in our study) [5]. Referring to the simplified TPA approach for estimating highfrequency component of heart rate variability proposed by Yokoyama et al., Sugiura et al. described the relationship between TPA calculated using pulse waves which are far easier to measure than a heart rate and subjective stress. While these schemes involving collection of saliva and measurement of heart rate may be fine for the laboratory, they would be hard to implement in an everyday life setting such as we propose. The pulse-wave approach is similar to our idea in that it involves a simple device, but it fails as a practical solution for a couple of reasons: pulse waves are not all that accurate, and the sensor is attached to the subject's fingertip, which would interfere with the subject's daily activities.

As one can see from the photo in Fig.1, the NeuroSky MindSet neuroheadset resembles a pair of headphones, so the user has total freedom of movement when wearing the device. Compared to conventional electroencephalography (EEG) recording devices that use multiple "wet" electrodes placed on a subject's scalp, the Mindset headset features a single "dry" electrode-fitted arm that contacts with the user's forehead, transmits data via wireless Bluetooth, and is well suited to mobile applications. Mindset was marketed as a hobby or entertainment device, and largely overlooked for serious medical or scientific applications. Yet despite its simple specifications, the Mindset has proved effective as a SSVEPbased brain-computer interface [6], and been used to estimate stress in many studies [7], [8], [9]. It has been proven that a dry electrode has the ability to retrieve EEG power spectra extremely similar to the one acquired from a wet electrode [10], and has demonstrated potential as an interface. The Mindset should work sufficiently well for the purposes of our project in estimating subjective assessment. In this study, we assess our ability to estimate subjective assessments using objective biosignals captured by the Mindset EEG headset (512 Hz sampling rate). The Mindset can acquire eight kinds of biosignals, $\delta, \theta, \alpha_{\mathrm{h}}, \alpha_{\mathrm{l}}, \beta_{\mathrm{h}}, \beta_{\mathrm{l}}, \gamma_{\mathrm{h}}$ and $\gamma_{\mathrm{l}}$. Each biosignal has different frequency: $\delta$ is from $0.5 \mathrm{~Hz}$ to $3 \mathrm{~Hz}, \theta$ is from $3 \mathrm{~Hz}$ to $8 \mathrm{~Hz}, \alpha_{\mathrm{h}}$ is from $10 \mathrm{~Hz}$ to $12 \mathrm{~Hz}, \alpha_{1}$ is from $8 \mathrm{~Hz}$ to $10 \mathrm{~Hz}, \beta_{\mathrm{h}}$ is from $20 \mathrm{~Hz}$ to $30 \mathrm{~Hz}, \beta_{\mathrm{l}}$ is from $12 \mathrm{~Hz}$ to $20 \mathrm{~Hz}$, $\gamma_{\mathrm{h}}$ is from $45 \mathrm{~Hz}$ to $60 \mathrm{~Hz}$, and $\gamma_{\mathrm{l}}$ is from $30 \mathrm{~Hz}$ to $45 \mathrm{~Hz}$. Our findings suggest that, at least to a certain extent, it is indeed possible to estimate subjective assessment of stress using the Mindset neuroheadset.

\section{EXPERIMENT}

We organized an experiment to evaluate the ability of the simple Mindset neuroheadset to estimate subjective human assessments. The experiment was designed to stimulate psychological state changes in the subjects by intermittently inducing stress in the subjects.

\section{A. Methodology}

The subjects were 16 young men all in their 20s, and the experiment was conducted in a closed, quiet room. Prior to the experiment, the subjects given a brief explanation of the experiment and allowed to practice for one minute the type of mental arithmetic task that would be used to induce psychological stress. The subjects were then seated, they wore the MindSet headsets, and began the experiment. Fig.2 is a time chart showing the sequence of the experiment. The trial consists of sets that are done sequentially with each set consisting to two phases: a five-minute rest state $\left(\mathrm{R}_{\mathrm{i}}\right)$ where the subject rest quietly with eyes closed followed by a five-minute stress state $\left(\mathrm{S}_{\mathrm{i}}\right)$ where the subjects tackle the mental arithmetic task. The experiment consists of five of these sets that are done sequentially $(i=1, \ldots, 5)$, and the entire experiment lasts about 50 minutes. Throughout the trial, the subjects are queried about their current psychological states immediately after each rest and stress state (at times $\mathrm{T}_{1}, \ldots, \mathrm{T}_{10}$ in Fig.2), and their responses are used as the subjects' own subjective assessments. At the same time, the subjects' biosignals were recorded by the Mindset all through the experiment. Each subject was subjected to one round of the intermittent procedure outlined in Fig.2.

\section{B. Psychological Stress Test}

We induced psychological stress in the subjects by having them do math calculations in their heads. The subjects were asked to count down from a large 4-digit prime number in increments of 13 as quickly as possible. The subjects did the 


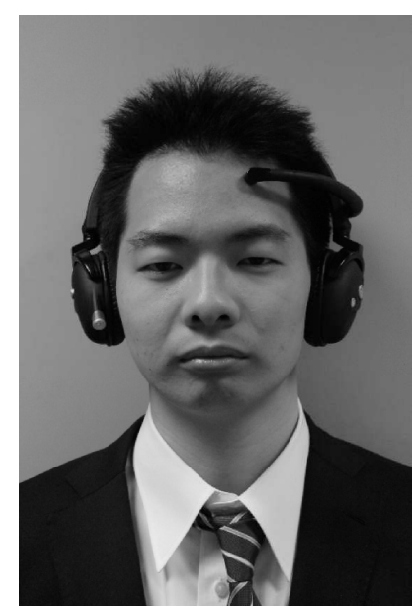

Fig. 1. NeuroSky MindSet

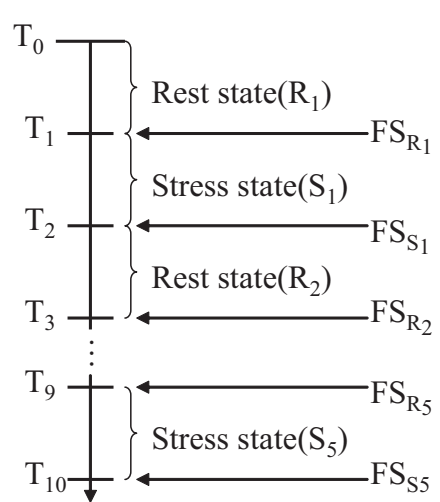

time[sec]

Fig. 2. Experimental time chart

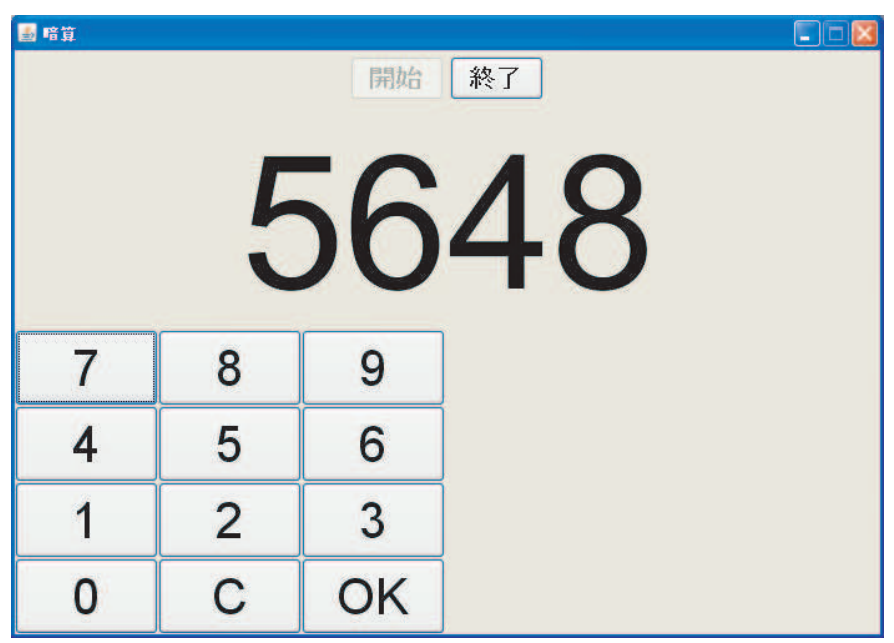

Fig. 3. Mental arithmetic answer application

subtraction in their heads by manipulating the mouse using the mental arithmetic answer application displayed on their PC screens as shown in Fig.3. The first 4-digit number is displayed at random, and if the subject answers incorrectly, he has to go back to the beginning number. Subjects continue counting down in increments of 13 , and if the number is reduced to less than 13, a new 4-digit prime number is selected at random and the subject continues counting down. This mental arithmetic task was adopted from "The Trier Social Stress Test: A Tool for Investigating Psychobiological Stress Responses in a Laboratory Setting" [11], and the idea of subjecting subjects to five rounds of mental math tasks was taken from "The Variations of Tenseness and Heart Rate During the Repeated Mental Arithmetic Tasks" [12].

\section{Subjective Assessment by Face Scale}

We used the face scale as an indicator of the subjects' subjective assessment of their psychological states. Proposed by Lorish et al. [13], the face scale is a subjective assessment scheme consisting of 20 drawings of a single face, with


6

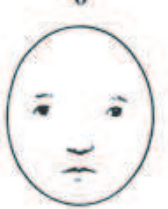

11

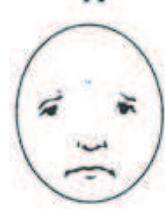

16
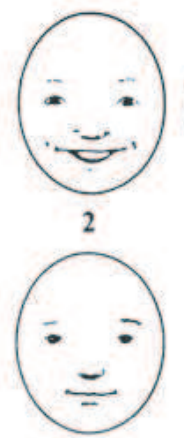

7

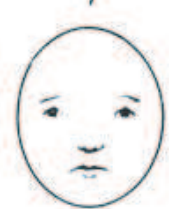

12

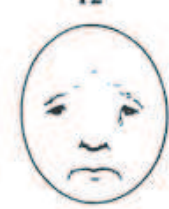

17

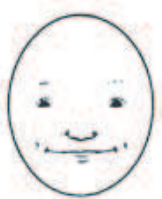

3

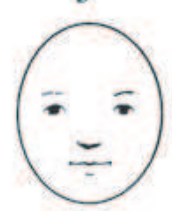

8

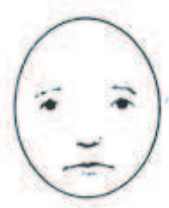

13

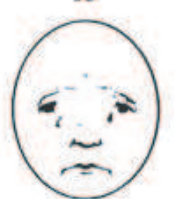

18

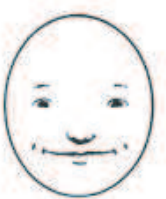

4

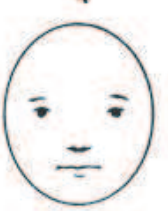

9


19

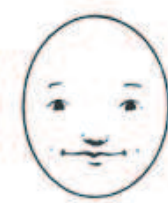

5

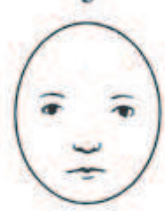

10

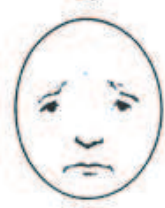

15

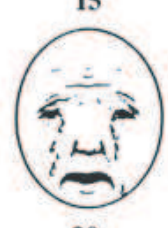

20
Fig. 4. Face Scale

each face depicting a slightly different mood state numbered from 1 to 20, with 1 representing the most positive mood (a very happy face) and 20 representing the most negative mood (a very sad face). Subjects can very quickly and easily subjectively assess the way they are feeling throughout the experiment by simply indicating the face that best reflects how they are feeling at the moment. The subjects were instructed to choose the face from the face scale that best represented their mood or how they were feeling at the moment. Fig.4 shows the face scale developed by Lorish et al. that was used in this work.

\section{Biosignals and Face Scale}

Here we assume that the brief intervals just before the end of each state shown in Fig.2 are the optimum times for identifying the states. In other words, the biosignal reading at $\tau$ seconds just before time $T_{1}$ at the end of the first rest state $R_{1}$ is defined as $\overline{\mathrm{X}}_{\mathrm{R}_{1}(\tau)} \cdot \overline{\mathrm{X}}_{\mathrm{R}_{1}(\tau)}$ indicates the average of $\mathrm{X}_{\mathrm{R}_{1}(\tau)}(\mathrm{t})$ that is a biosignal recorded at the time $t\left(=T_{1}-\tau, \ldots, T_{1}\right)$. Similarly, the biosignal at $\tau$ seconds just before time $\mathrm{T}_{2}$ at the end of the first mental arithmetic-induced stress state is assumed to be $\overline{\mathrm{X}}_{\mathrm{S}_{1}(\tau)}$. In other words, the biosignal of the $n$-th rest state $R_{n}$ can be written $\bar{X}_{R_{n}(\tau)}$, and the biosignal of the $m$-th stress state $S_{m}$ is given as $\bar{X}_{S_{m}(\tau)}$. For the purposes of this work, we define $\tau$ as 30 seconds. Moreover, eight kinds of biosignals, $\mathbf{M}_{\mathbf{8}}=\left\{\delta, \theta, \alpha_{\mathrm{h}}, \alpha_{\mathrm{l}}, \beta_{\mathrm{h}}, \beta_{\mathrm{l}}, \gamma_{\mathrm{h}}, \gamma_{\mathrm{l}}\right\}$, are obtained from the MindSet sensor at a frequency of once per second.

We consider the feasibility of estimating the subjects' subjective face scale assessments based solely on biosignals 
derived from the Neurosky Mindset.

First we should note there are bound to be differences among the subjects in the way these states are felt or perceived, so rather than using absolute biosignal amounts, we will use the amounts of biosignal change for our evaluation. As a matter of fact, before deciding to use the change, we did attempt to evaluate estimations of subjective assessments using absolute biosignal amounts, but no correlations were observed. This can be explained as follows. In the initial rest state $R_{1}$, all subjects start from the same initial rest state environment with their eyes closed. Yet there is no way to control for individual differences among the subjects, so there was no way to set initial subjective assessment or biosignal values at the same starting point. In fact, the subjective assessments and biosignals obtained in our experiment were inconsistent at the initial state. This is why no correlations were found when we used absolute biosignal amounts, and why we made our evaluations based strictly on amount of change of each evaluation value. Following this reasoning, we derived the amount of change using the difference between $\mathrm{X}_{\mathrm{R}_{1}(\tau)}$ as the initial rest state of the first rest state $R_{1}$ and the average $\overline{\mathrm{X}}_{\mathrm{R}_{1}(\tau)}$. Here, the average amount of change in each state is defined as $\Delta \overline{\mathrm{X}}_{\mathrm{R}_{\mathrm{n}}(\tau)}, \Delta \overline{\mathrm{X}}_{\mathrm{S}_{\mathrm{m}}(\tau)}$, and our evaluations are based on these values.

$$
\begin{aligned}
\Delta \overline{\mathrm{X}}_{\mathrm{R}_{\mathrm{n}}}= & \frac{1}{\tau} \int_{\left(\mathrm{T}_{2 \mathrm{n}-1}-\tau\right)}^{\mathrm{T}_{2 \mathrm{n}-1}}\left\{\mathrm{X}_{\mathrm{R}_{\mathrm{n}}(\tau)}(\mathrm{t})-\overline{\mathrm{X}}_{\mathrm{R}_{1}(\tau)}\right\} \mathrm{dt} \\
\Delta \overline{\mathrm{X}}_{\mathrm{S}_{\mathrm{m}}}= & \frac{1}{\tau} \int_{\left(\mathrm{T}_{2 \mathrm{~m}}-\tau\right)}^{\mathrm{T}_{2 \mathrm{~m}}}\left\{\mathrm{X}_{\mathrm{S}_{\mathrm{m}}(\tau)}(\mathrm{t})-\overline{\mathrm{X}}_{\mathrm{R}_{1}(\tau)}\right\} \mathrm{dt} \\
& \mathrm{X} \in\left\{\delta, \theta, \alpha_{\mathrm{h}}, \alpha_{1}, \beta_{\mathrm{h}}, \beta_{1}, \gamma_{\mathrm{h}}, \gamma_{1}\right\}
\end{aligned}
$$

Now turning to the face scale, we define the face scale assessment value at time $T_{1}$ at the end of the first rest state as $\mathrm{FS}_{\mathrm{R}_{1}}$, the face scale value at time $\mathrm{T}_{2}$ at the end of the first stress state as $\mathrm{FS}_{\mathrm{S}_{1}}$, the face scale value after the $\mathrm{n}$-th rest state as $\mathrm{FS}_{\mathrm{R}_{\mathrm{n}}}$, and the face scale value after the $\mathrm{m}$-th stress state as $\mathrm{FS}_{\mathrm{S}_{\mathrm{m}}}$. FS value indicates the number of face scale under the each face mark in Fig.4 . As described earlier for the biosignals, here too we used differences based on values at the end of the initial rest state.

$$
\begin{aligned}
\Delta \mathrm{FS}_{\mathrm{R}_{\mathrm{n}}} & =\mathrm{FS}_{\mathrm{R}_{\mathrm{n}}}-\mathrm{FS}_{\mathrm{R}_{1}} \\
\Delta \mathrm{FS}_{\mathrm{S}_{\mathrm{m}}} & =\mathrm{FS}_{\mathrm{S}_{\mathrm{m}}}-\mathrm{FS}_{\mathrm{R}_{1}}
\end{aligned}
$$

Setting the amount of change as shown above, we then performed a multiple regression analysis taking $\Delta \overline{\mathrm{X}}\left(\Delta \overline{\mathrm{X}}_{\mathrm{R}_{\mathrm{n}}(\tau)}\right.$ and $\left.\Delta \overline{\mathrm{X}}_{\mathrm{S}_{\mathrm{m}}(\tau)}\right)$ as explanatory variables, and $\Delta \mathrm{FS}\left(\Delta \mathrm{FS}_{\mathrm{R}_{\mathrm{n}}}\right.$ and $\Delta \mathrm{FS}_{\mathrm{S}_{\mathrm{m}}}$ ) as corresponding criterion variables. Based on the defining equations for amounts of change given above, the amount of change for each biosignal can be written $\Delta \overline{\mathbf{M}}_{8}=\left\{\Delta \bar{\delta}, \Delta \bar{\theta}, \Delta \bar{\alpha}_{\mathrm{h}}, \Delta \bar{\alpha}_{1}, \Delta \bar{\beta}_{\mathrm{h}}, \Delta \bar{\beta}_{1}, \Delta \bar{\gamma}_{\mathrm{h}}, \Delta \bar{\gamma}_{1}\right\}$

\section{EXPERIMENTAL RESULTS AND DISCUSSION}

\section{A. Assessment by Multiple Regression Analysis}

Then, performing a multiple regression analysis for all eight dimensions of the biosignals using $\Delta \overline{\mathbf{M}}_{\mathbf{8}}$ as an explanatory variable, we obtain the coefficient correlation shown in Table I.

TABLE I

COEFFICIENT CORRELATION

\begin{tabular}{cc}
\hline Explanatory variable & Coefficient correlation \\
\hline$\Delta \overline{\mathbf{M}}_{\mathbf{8}}$ & 0.5609 \\
\hline
\end{tabular}

A coefficient correlation of 0.5609 for an explanatory variable of $\Delta \overline{\mathbf{M}}_{\mathbf{8}}$ reveals that there is some degree of correlation. While we should be able to estimate changes in human subjective assessments to some degree, it is clear that the estimation accuracy is insufficient. Moreover, the fact that we are dealing with amounts of change means that we need to know the initial biosignal and subjective assessment states, so there are clearly some practical issues still to be resolved.

When multiple regression analysis involves a large number of variable, usually some process is used to select variables. One approach is to combine the variables into groups then perform multiple regression analysis on all the combinations, but the correlation coefficient doesn't change much no matter how the variables are combined. By way of reference, Table II shows the correlation coefficients when correlations are derived for $\Delta \mathrm{FS}$ using $\Delta \mathrm{FS}$ and amount of biosignal change as the explanatory variables. Among these values, Table II shows $\Delta \bar{\beta}_{\mathrm{h}}$ having the largest absolute value correlation coefficient, and Fig.5 shows the relation between $\Delta \bar{\beta}_{\mathrm{h}}$ and $\Delta \mathrm{FS}$.

\section{B. Assessment by Discriminant Analysis}

Based on finding of the previous section, here again we reset the subjects' two states (rest and stress) that we want to estimate from the biosignals. Again, our objective is to explore the feasibility of estimating subjective assessments (face scale moods) based on the results of state estimates. For these estimates, we use absolute amounts and not the change amounts used in the previous section. As an example, Fig.6 shows a scatter plot and discriminant line for the two variables in Table II having the highest absolute values. The line drawn in the figure is the discriminate line derived by linear discriminant analysis. While only two variables are used, the discriminate line nevertheless enables us to visualize the different state categories to some degree.

Even though only two variables are used in Fig.6, here again we apply discriminant analysis to the problem of sorting or classifying these two states by eight dimensional $\mathbf{M}_{\mathbf{8}}$ data. Table III summarizes discriminant analysis results for each subject taking the moving average of biosignals from the Mindset sensor in five-second window intervals as the explanatory variable and the two states (rest and stress) as the criterion variable. 


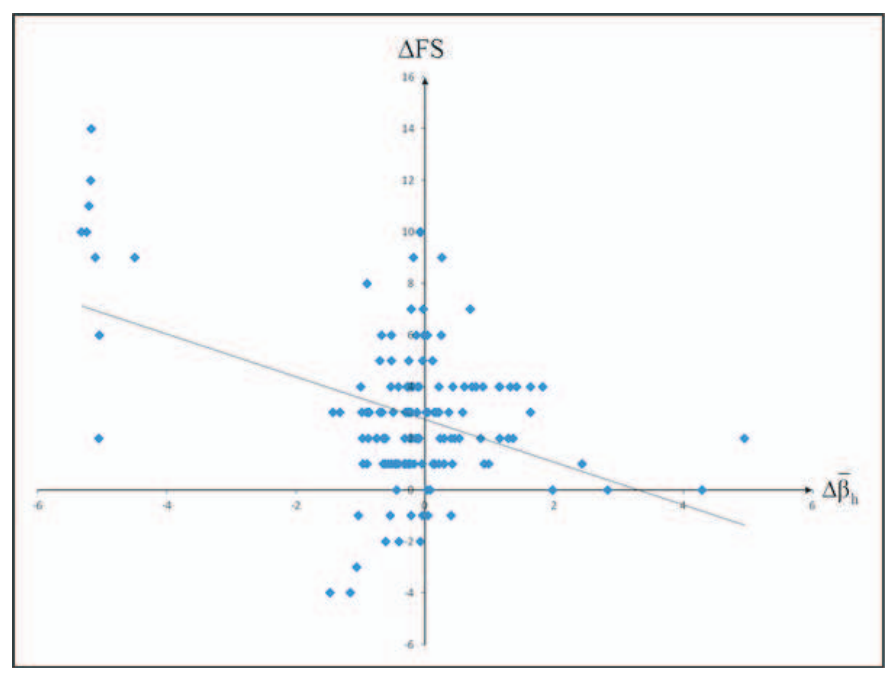

Fig. 5. $\Delta \bar{\beta}_{\mathrm{h}}$ and $\Delta \mathrm{FS}$ correlation diagram

The average discriminant rate for the 16 subjects was $83.08 \%$, thus clearly showing that state discrimination by Neurosky MindSet is quite accurate. Note that nine of the 16 subjects had discriminant rates exceeding $80 \%$-and of those, four subjects had discriminant rates above $90 \%$-so it is clear that this approach yields very high state discrimination.

TABLE II

$\triangle$ FS AND COEFFICIENT CORRELATION

\begin{tabular}{lc}
\hline Variable & Coefficient correlation \\
\hline$\Delta \bar{\delta}$ & -0.2592 \\
$\Delta \bar{\theta}$ & -0.3107 \\
$\Delta \bar{\alpha}_{\mathrm{h}}$ & -0.2475 \\
$\Delta \bar{\alpha}_{1}$ & -0.3180 \\
$\Delta \bar{\beta}_{\mathrm{h}}$ & -0.4249 \\
$\Delta \bar{\beta}_{1}$ & -0.1457 \\
$\Delta \bar{\gamma}_{\mathrm{h}}$ & -0.1026 \\
$\Delta \bar{\gamma}_{1}$ & -0.1346 \\
\hline
\end{tabular}

Let us next consider the relationship between the discriminant analysis results and the subjective assessments. First we describe the subjective assessments of the original rest and stress states before the discriminant analysis was performed. As one can see in Fig.7, a significant difference level of $1 \%(\mathrm{P}=0.0000)$ was obtained by Wilcoxon signed rank sum test using face scale data obtained from all the subjects between the group of subjective assessments in the rest state and the group of subjective assessments in the stress state. This tells us that subjective assessments of the subjects are changed significantly by the stress-inducing mental arithmetic task.

Next we turn to subjective assessments of the each group that is determined by the discriminant analysis to be either in a state of rest or in a state of stress. Of course, these results are not $100 \%$ accurate, so there are bound to be some mix-ups and false positives. Using a Mann-Whitney $U$ test we again found, as illustrated in Fig.8, a significant difference level of $1 \%(\mathrm{P}=0.0010)$ between the group of subjective assessments

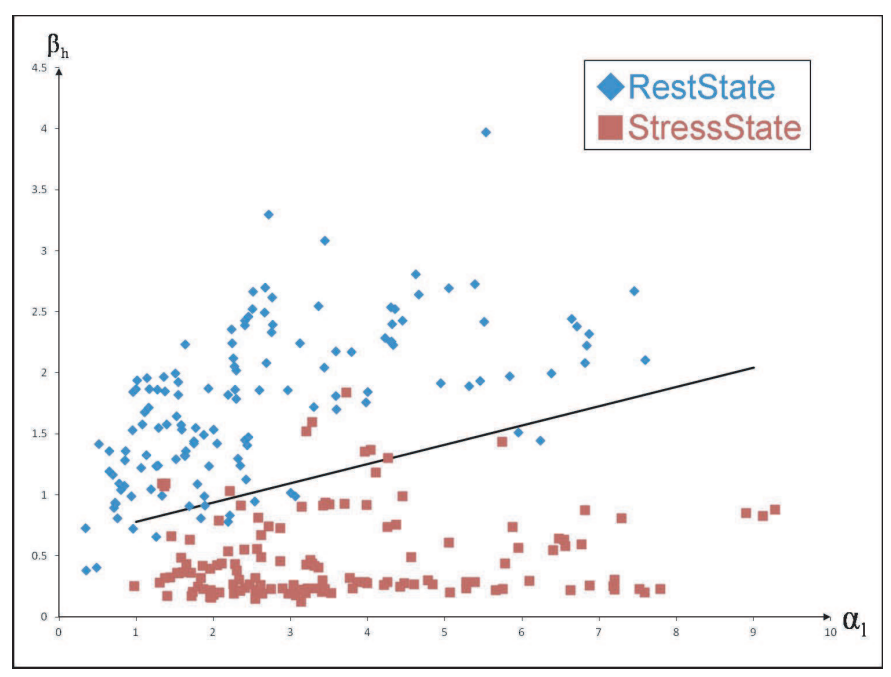

Fig. 6. Discriminant analysis for $\alpha_{1}$ and $\beta_{\mathrm{h}}$

TABLE III

DISCRIMINANT RATE

\begin{tabular}{lc}
\hline & Discriminant rate \\
\hline Subject A & $98.52 \%$ \\
Subject B & $91.85 \%$ \\
Subject C & $75.19 \%$ \\
Subject D & $76.30 \%$ \\
Subject E & $68.52 \%$ \\
Subject F & $85.19 \%$ \\
Subject G & $88.15 \%$ \\
Subject H & $77.41 \%$ \\
Subject I & $81.48 \%$ \\
Subject J & $77.04 \%$ \\
Subject K & $74.07 \%$ \\
Subject L & $84.07 \%$ \\
Subject M & $83.33 \%$ \\
Subject N & $97.04 \%$ \\
Subject O & $76.67 \%$ \\
Subject P & $94.44 \%$ \\
\hline Average & $83.08 \%$ \\
\hline
\end{tabular}

judged to be in a state of rest versus the group of subjective assessments judged to be in a state of stress.

Note that these two tests are used differently. The Wilcoxon signed rank sum test is used to assess correspondence when the number of subjective assessments for the rest state and the stress state are the same, while the Mann-Whitney U test uses assessments that are determined by discriminant analysis to be either in a state of rest or stress, so there is no guaranteeing that the number of subjective assessments for each state will be the same or that they will correspond. Based on the results of these tests, we should expect the discriminant analysis to yield an approximate estimation of human subjective assessments.

\section{CONClusions}

This study examined the relationship between groups of biosignals obtained using a simple Neurosky Mindset neuroheadset as objective observable data and face scale subjective assessments. Focusing on the amount of biosignal change 


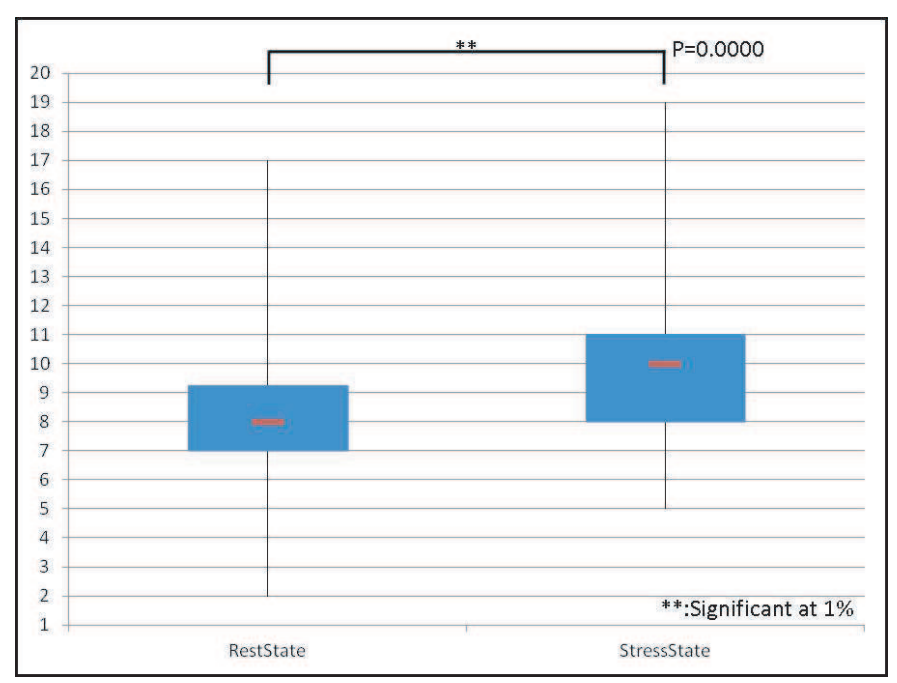

Fig. 7. FS distribution charts for rest and stress states

using multiple regression analysis, we found that the face scale assessments were correlated to some degree, but the results were not sufficiently accurate. However, using discriminant analysis to differentiate mental states of subjects, we obtained an impressively accurate average discriminant rate of $83.08 \%$, with rates for some of the subjects even exceeding $90 \%$. This approach has excellent potential for achieving a very high rate of state discrimination accuracy. We also examined subjective assessments based on state discrimination results, and found a significant difference in subjective assessments between the two states. In this case, the estimation of subjective assessment was done as a normal distribution with respect to the absolute amount of the face scale that has a constant distribution.

This experiment reveals that, even using a device as simple as the Mindset neuroheadset, this approach shows definite potential as a tool for estimating emotional and psychological states of humans. Especially when estimating subjective assessments and when estimating states from absolute amounts of $8 \mathrm{D}$ biosignals, we obtained remarkably accurate results. By using absolute amounts of biosignals rather than amounts of change, we become less dependent on individual differences and initial states. This is a major advantage of wearing biosensors and capturing biosignals throughout the day, the approach that we advocate here.

This experiment was designed to control subjects by applying stress at regular intervals. Here we used a mental arithmetic task to induce stress, but further research is clearly required that involves other sources of stress and experimental designs that better replicate real life situations.

\section{REFERENCES}

[1] R. W. Picard, Affective Computing. The MIT Press, 1997.

[2] Rafael A. Calvo, Sidney D'Mello, "Affect Detection: An Interdisciplinary Review of Models, Methods, and Their Applications", IEEE Transactions on Affective Computing, Vol.1, No.1, pp. 18-37, 2010.

[3] S. Nomura, H. Tanaka, T. Mizuno, A. Nozawa, T. Nagashima, H. Ide, "Evaluation of the Effect of Web Surfing on Human: Introducing Human



Fig. 8. Rest and stress state FS distributions determined by discriminant analysis

Secretary Immune and Endocrine Indices," the IEICE Transactions on Information and Systems, J91-D(4), pp. 1158-1167, April 1, 2008.

[4] K. Yokoyama, N. Kamiya, "Simplified method for estimating highfrequency component of heart rate variability based on local characteristics in time series," The Japanese Journal of Ergonomics, Vol. 40, No. 6, pp. 302-308, Dec. 15, 2004.

[5] T. Sugiura, Y. Okudaira, T. Nakamura, H. Itoh, "Relaxation Effects of Object Speed in Video Images: Evaluation by Heart-Rate Variability Analysis," Transactions of Japan Society of Kansei Engineers, Vol. 8, No. 3, pp. 595-602, 2009.

[6] A. Luo, T. J. Sullivan, "A user-friendly SSVEP-based brain computer interface using a time-domain classifier", Journal of Neural Engineering, Vol. 7, No. 2, pp. 026010, 2010.

[7] G. Rebolledo-Mendez, I. Dunwell, E. A. Martinez-Miron, M. D. VargasCerdan, S. Freitas, F. Liarokapis, A. R. Garcia-Gaona: "Assessing NeuroSky's Usability to Detect Attention Levels in an Assessment Exercise," Proceedings of the 13th International Conference on Human-Computer Interaction, Part I, HCII 2009, LNCS 5610, pp. 149-158, 2009.

[8] Y. Yasui, "A Brainwave Signal Measurement and Data Processing Technique for Daily Life Applications," Journal of Physiological Anthropology, Vol. 28, No. 3, pp. 145-150, May 2009.

[9] E. Haapalainen, S. J. Kim, J. F. Forlizzi, A. K. Dey, "Psycho-Physiological Measures for Assessing Cognitive Load," Proceedings of the 12th ACM International Conference on Ubiquitous Computing, Ubicomp '10, pp. 301-310, 2010.

[10] J. C. Chiou, Li-Wei Ko, Chin-Teng Lin, Chao-Ting Hong, Tzyy-Ping Jung, "Using Novel MEMS EEG Sensors in Detecting Drowsiness Application," IEEE Biomedical Circuits and Systems Conference, 2006.

[11] C. Kirschbaum, K. M. Pirke, D. H. Hellhammer, "The 'Trier Social Stress Test'-a tool for investigating psychobiological stress responses in a laboratory setting," Neuropsychobiology, Vol. 28, pp. 76-81, 1993.

[12] M. Nagamine, M. Murota, M. Ishii, Y. Shimizu, "The Variations of Tenseness and Heart Rate During the Repeated Mental Arithmetic Tasks," Japan Journal of Educational Technology, Vol. 25, No. 4, pp. 237-246, March 20, 2002.

[13] C. D. Lorish, R. Maisiak: "The face scale: a brief, nonverbal method for assessing patient mood," Arthritis \& Rheumatism, Vol. 29, Issue 7 , pp. 906-909, July 1986. 